\title{
A CHARACTERIZATION OF TWO WEIGHT NORM INEQUALITIES FOR FRACTIONAL AND POISSON INTEGRALS
}

\author{
ERIC T. SAWYER
}

ABSTRACT. For $1<p \leq q<\infty$ and $w(x), v(x)$ nonnegative functions on $\mathbf{R}^{n}$, we show that the weighted inequality

$$
\left(\int|T f|^{q} w\right)^{1 / q} \leq C\left(\int f^{p} v\right)^{1 / p}
$$

holds for all $f \geq 0$ if and only if both

$$
\int\left[T\left(\chi Q v^{1-p^{\prime}}\right)\right]^{q} w \leq C_{1}\left(\int_{Q} v^{1-p^{\prime}}\right)^{q / p}<\infty
$$

and

$$
\int[T(\chi Q w)]^{p^{\prime}} v^{1-p^{\prime}} \leq C_{2}\left(\int_{Q} w\right)^{p^{\prime} / q^{\prime}}<\infty
$$

hold for all dyadic cubes $Q$. Here $T$ denotes a fractional integral or, more generally, a convolution operator whose kernel $K$ is a positive lower semicontinuous radial function decreasing in $|x|$ and satisfying $K(x) \leq C K(2 x), x \in \mathbf{R}^{n}$. Applications to degenerate elliptic differential operators are indicated.

In addition, a corresponding characterization of those weights $v$ on $\mathbf{R}^{n}$ and $w$ on $\mathbf{R}_{+}^{n+1}$ for which the Poisson operator is bounded from $L^{p}(v)$ to $L^{q}(w)$ is given.

1. Introduction. Suppose $1 \leq p, q \leq \infty, v(x)$ and $w(x)$ are nonnegative measurable functions (i.e. weights) on $\mathbf{R}^{n}$ and $\mathbf{R}^{m}$ respectively, and that $T$ is an operator taking suitable functions on $\mathbf{R}^{n}$ into functions on $\mathbf{R}^{m}$. In his survey article [26], B. Muckenhoupt raised the general question of characterizing when the weighted norm inequality,

$$
\left(\int_{\mathbf{R}^{m}}|T f(x)|^{q} w(x) d x\right)^{1 / q} \leq C\left(\int_{\mathbf{R}^{n}}|f(x)|^{p} v(x) d x\right)^{1 / p},
$$

holds for all appropriate $f$. In the case of "one weight", e.g. $p=q, m=n$ and $w=v$, and for many classical operators $T$, inequality (1.1) can be characterized by remarkably simple conditions, most notable being that the $A_{p}$ condition,

$$
\left(A_{p}\right) \quad\left(\frac{1}{|Q|} \int_{Q} w\right)\left(\frac{1}{|Q|} \int_{Q} w^{1-p^{\prime}}\right)^{p-1} \leq C \quad \text { for all cubes } Q \subset \mathbf{R}^{n} \text {, }
$$

Received by the editors August 1, 1986.

1980 Mathematics Subject Classification (1985 Revision). Primary 42B25.

Research supported in part by NSERC grant A5149. 
is necessary and sufficient for the Hardy-Littlewood maximal function and Hilbert transform inequalities (see [19 and 25]).

The case of different weights has been far less accommodating. Only for the simplest of nontrivial operators, namely the Hardy operator, $T f(x)=\int_{0}^{x} f(t) d t$, has a correspondingly simple characterizing condition for (1.1) been obtained (see $[1,3,25,40$ and 41]), namely

$$
\left(\int_{r}^{\infty} w\right)^{1 / q}\left(\int_{0}^{r} v^{1-p^{\prime}}\right)^{1 / p^{\prime}} \leq C \text { for all } 0<r<\infty .
$$

In a sense, the next simplest classical operator is the Hardy-Littlewood maximal function,

$$
M f(x)=\sup _{x \in Q \text { cube }} \frac{1}{|Q|} \int_{Q}|f|,
$$

and in [31] it was shown that for $T=M,(1.1)$ holds if and only if

$$
\left(\int_{Q}\left[M\left(\chi_{Q} v^{1-p^{\prime}}\right)\right]^{q} w\right)^{1 / q} \leq C\left(\int_{Q} v^{1-p^{\prime}}\right)^{1 / p}<\infty \quad \text { for all cubes } Q \subset \mathbf{R}^{n} .
$$

In particular, this says that (1.1), with $T=M$, holds for all $f$ if it holds when tested over functions of the form $f=\chi_{Q} v^{1-p^{\prime}}$ (since then $f^{p} v=f$ ). This suggested a reasonable conjecture: (1.1) holds for all $f$ provided it holds when tested over functions of the form $\chi_{Q} v^{1-p^{\prime}}$, where the sets $Q$ are appropriately related to the geometry of the operator $T$. While this is born out in the one weight cases considered above and in the two weight inequality for $M$, it fails, for example, for fractional integrals,

$$
T f(x)=I_{\alpha} f(x)=\int_{\mathbf{R}^{n}}|x-y|^{\alpha-n} f(y) d y,
$$

and for higher dimensional Hardy operators

$$
T f\left(x_{1}, \ldots, x_{n}\right)=\int_{0}^{x_{1}} \cdots \int_{0}^{x_{n}} f\left(t_{1}, \ldots, t_{n}\right) d t_{1} \cdots d t_{n}
$$

(see [32] and [33] respectively for counterexamples). The point here, first indicated in the work of B. Muckenhoupt and R. L. Wheeden in [28], is that for linear operators, one should also test the inequality dual to (1.1) over appropriate test functions.

It is convenient at this point to recast (1.1) in a more "natural" form, one that permits the replacement of the functions $v$ and $w$ by positive Borel measures $\mu$ and $\omega$, and that leads more naturally to the correct testing functions:

$$
\left(\int_{\mathbf{R}^{m}}|T(f \mu)(x)|^{q} d \omega(x)\right)^{1 / q} \leq C\left(\int_{\mathbf{R}^{n}}|f(x)|^{p} d \mu(x)\right)^{1 / p}, \quad f \in L^{p}(\mu) .
$$

To see that (1.1) is included in (1.2), set $d \omega(x)=w(x) d x, d \mu(x)=v(x)^{1-p^{\prime}} d x$ and replace $f$ by $f v^{p^{\prime}-1}$ in (1.2). If $T$ is linear and $T^{*}$ its dual under the usual pairing, i.e.

$$
\int_{\mathbf{R}^{m}}(T f)(x) g(x) d x=\int_{\mathbf{R}^{n}} f(y)\left(T^{*} g\right)(y) d y \quad \text { for all } f \text { and } g,
$$


then (1.2) is equivalent to the dual inequality,

$$
\left(\int_{\mathbf{R}^{n}}\left|T^{*}(g \omega)(x)\right|^{p^{\prime}} d \mu(x)\right)^{1 / p^{\prime}} \leq C\left(\int_{\mathbf{R}^{m}}|g(x)|^{q^{\prime}} d \omega(x)\right)^{1 / q^{\prime}}, \quad g \in L^{q^{\prime}}(\omega) .
$$

The new conjecture is that (1.2) holds for all $f$ in $L^{p}(\mu)$ if and only if both (1.2) and (1.3) hold when tested over characteristic functions of sets $Q$ appropriately related to the geometry of $T$. In [33], this conjecture was established for the twodimensional Hardy operator

$$
T f\left(x_{1}, x_{2}\right)=\int_{0}^{x_{1}} \int_{0}^{x_{2}} f\left(t_{1}, t_{2}\right) d t_{1} d t_{2}
$$

by showing that it sufficed to test (1.2) over rectangles of the form $[0, a] \times[0, b]$ and to test (1.3) over rectangles of the form $[a, \infty] \times[b, \infty]$.

The purpose of this paper is to establish the conjecture above for fractional integral operators (and some generalizations thereof) along with the Poisson integral operator. For other work on weighted inequalities for these operators, see $[\mathbf{2}, \mathbf{4 , 6}$, $7,9,10,11,15,17,20,21,22,23,27,30,32,35,36,39]$ and references given there. Before stating our two theorems, we establish some notation. Given a cube $Q$ and $R>0$, denote by $R Q$ the cube concentric with $Q$ and with $R$ times the side length. For any measure $\mu$ and set $E$, denote by $|E|_{\mu}$ the $\mu$-measure of $E$. Finally, the letter $C$ will be used to denote a positive constant that may change from line to line but will remain independent of the appropriate quantities.

THEOREM 1. Suppose $1<p \leq q<\infty$, $\omega$ and $\mu$ are positive Borel measures on $\mathbf{R}^{n}$, and $T f=K * f$ where $K(x)$ is a positive lower semicontinuous radial function decreasing in $|x|$ and satisfying the growth condition $K(x) \leq C K(2 x), x \in \mathbf{R}^{n}$.

Then the weighted inequality

$$
\left(\int[T(f \mu)]^{q} d \omega\right)^{1 / q} \leq C\left(\int f^{p} d \mu\right)^{1 / p} \quad \text { for all } f \geq 0
$$

holds if and only if both

$$
\left(\int\left[T\left(\chi_{Q} \mu\right)\right]^{q} d \omega\right)^{1 / q} \leq C_{1}|Q|_{\mu}^{1 / p}<\infty \quad \text { for all dyadic cubes } Q
$$

and

$$
\left(\int\left[T\left(\chi_{Q} \omega\right)\right]^{p^{\prime}} d \mu\right)^{1 / p^{\prime}} \leq C_{2}|Q|_{\omega}^{1 / q^{\prime}}<\infty \quad \text { for all dyadic cubes } Q .
$$

THEOREM 2. Suppose $1<p \leq q<\infty, \omega$ and $\mu$ are positive Borel measures on $\mathbf{R}_{+}^{n+1}$ and $\mathbf{R}^{n}$ respectively, and

$$
\begin{aligned}
& P(f \mu)(x, t)=\int_{\mathbf{R}^{n}} P_{t}(x-y) f(y) d \mu(y), \\
& P^{*}(g \omega)(y)=\int_{\mathbf{R}_{+}^{n+1}} P_{t}(y-x) g(x, t) d \omega(x, t),
\end{aligned}
$$


denote the Poisson and dual Poisson operators. Then the weighted inequality

$$
\left(\int_{\mathbf{R}_{+}^{n+1}}[P(f \mu)]^{q} d \omega\right)^{1 / q} \leq C\left(\int_{\mathbf{R}^{n}} f^{p} d \mu\right)^{1 / p} \quad \text { for all } f \geq 0
$$

holds if and only if

$$
\left(\int_{\mathbf{R}_{+}^{n+1}}\left[P\left(\chi_{Q} \mu\right)\right]^{q} d \omega\right)^{1 / q} \leq C|Q|_{\mu}^{1 / p}<\infty \quad \text { for all dyadic cubes } Q \subset \mathbf{R}^{n}
$$

and

$$
\begin{array}{r}
{\left[\int_{\mathbf{R}^{n}}\left|P^{*}\left[\chi_{\hat{Q}}(x, t) t^{q-1} d \omega(x, t)\right]\right|^{p^{\prime}} d \mu\right]^{1 / p^{\prime}} \leq C\left(\int_{\hat{Q}} t^{q} d \omega(x, t)\right)^{1 / q^{\prime}}<\infty} \\
\quad \text { for all dyadic cubes } Q \subset \mathbf{R}^{n}
\end{array}
$$

where $\hat{Q}$ denotes the cube in $\mathbf{R}_{+}^{n+1}$ having $Q$ as a face.

Applications of Theorem 1 to regularity and eigenvalve estimates for degenerate elliptic differential operators are readily suggested by the following observation: Suppose $f$ has support in, or mean zero on, a cube $Q$. Then (see [12])

$$
|f(x)| \leq C I_{1}\left(\chi_{Q}|\nabla f|\right)(x), \quad x \in Q,
$$

where $I_{1}$ denotes the fractional integral of order 1 given by

$$
I_{1} f(x)=\int_{\mathbf{R}^{n}}|x-y|^{1-n} f(y) d y .
$$

Thus if $d \mu=\chi_{Q}(x) v(x)^{1-p^{\prime}} d x$ and $d \omega=\chi_{Q}(x) w(x) d x$ satisfy conditions (1.5) and (1.6) with $T=I_{1}$, then we have the two weight Poincaré-Sobolev inequality

$$
\int_{Q}|f(x)|^{2} w(x) d x \leq C_{Q} \int_{Q}|\nabla f(x)|^{2} v(x) d x
$$

for all $f$ with either supp $f \subset \stackrel{\circ}{Q}$ or $\int_{Q} f=0$, and where $C_{Q}$ is a fixed multiple of $C_{1}+C_{2}$. This inequality has been used by $\mathrm{S}$. Chanillo and $\mathrm{R}$. L. Wheeden [8] to study the local behaviour of solutions to degenerate elliptic operators $P=\nabla \cdot A \nabla$ where the weights $w(x)$ and $v(x)$ are given by the largest and smallest eigenvalues of $A(x)$ (see also [12, 18 and 38]). Inequality (1.11) is also the crucial ingredient in estimating eigenvalues for degenerate Schrödinger operators $-\nabla \cdot A \nabla+V$; see S. Chanillo and R. L. Wheeden [7] (see also [5, 14, 21 and 22] for earlier related results).

REMARK 1. Neither (1.5) nor (1.6) alone is in general sufficient for (1.4) as shown by the example in [32]. Note however that (1.5) and (1.6) coincide when $\omega=\mu$ and $q=p^{\prime}$.

REMARK 2. A modification of the proof of Theorem 1 given below shows that in conditions (1.5) and (1.6), the integrations on the left need be taken only over the cubes $Q$ and $12 Q$ respectively. We do not know if it is possible to restrict both integrations to $Q$. 
2. Proof of Theorem 1. Assume (1.4) holds. Then so does its dual inequality

$$
\left(\int[T(g \omega)]^{p^{\prime}} d \mu\right)^{1 / p^{\prime}} \leq C\left(\int g^{q^{\prime}} d \omega\right)^{1 / q^{\prime}} \quad \text { for all } g \geq 0 .
$$

With $f=\chi_{Q}$ in (1.4) and $g=\chi_{Q}$ in (2.1) we obtain (1.5) and (1.6).

Conversely, suppose (1.5) and (1.6) hold and, without loss of generality, that $f$ is nonnegative and bounded with compact support. Now $T(f \mu)$ is lower semicontinuous and so for each $k$, we can write the open set $\Omega_{k}=\left\{T(f \mu)>2^{k}\right\}$ as $\dot{\bigcup}_{j} Q_{j}^{k}$ where the $Q_{j}^{k}$ are the dyadic cubes maximal among those dyadic cubes $Q$ satisfying $R Q \subset \Omega_{k}$. Choosing $R \geq 3$ sufficiently large, depending only on the dimension $n$, we obtain

(i) (disjoint cover)

$$
\Omega_{k}=\bigcup_{j} Q_{j}^{k} \text { and } Q_{j}^{k} \cap Q_{i}^{k}=\varnothing \text { for } i \neq j,
$$

(ii) (Whitney condition) $R Q_{j}^{k} \subset \Omega_{k}$ and $3 R Q_{j}^{k} \cap \Omega_{k}^{c} \neq \varnothing$ for all $k, j$,

(iii)(finite overlap)

$$
\sum_{j} \chi_{3 Q_{j}^{k}} \leq C \chi_{\Omega_{k}} \text { for all } k
$$

(iv) (crowd control)

The number of cubes $Q_{s}^{k}$ intersecting a fixed cube $3 Q_{j}^{k}$ is at most $C$,

(v) (nested property) $\quad Q_{j}^{k} \subset Q_{i}^{l}$ implies $k \geq l$.

In fact, (i) and (v) are obvious, (ii) follows as in Theorem 2.1 of [16], and (iii) and (iv) are a consequence of (ii) and a geometric packing argument on p. 16 of [13].

We now claim the following maximum principle holds:

$$
T\left(\chi_{\left(3 Q_{j}^{k}\right)^{c}} f \mu\right)(x) \leq C 2^{k}, \quad x \in Q_{j}^{k},
$$

for all $(k, j)$ where $C$ is a constant. To see this, momentarily fix $(k, j)$ and choose $z \in 3 R Q_{j}^{k} \cap \Omega_{k}^{c}$, which is possible by the Whitney condition (2.2)(ii). From the growth assumption on $K(x)$, we conclude there is a constant $C$ such that $K(x-y) \leq C K(z-y)$ for $x \in Q_{j}^{k}, y \in\left(3 Q_{j}^{k}\right)^{c}$. Multiplying this inequality by $f(y)$ and then integrating over $\left(3 Q_{j}^{k}\right)^{c}$ with respect to $d \mu(y)$ yields $T\left(\chi_{\left(3 Q_{j}^{k}\right)^{c}} f \mu\right)(x) \leq$ $C T(f \mu)(z) \leq C 2^{k}$ since $z \notin \Omega_{k}$. This proves (2.3).

Now fix an integer $m \geq 2$ satisfying $2^{m-2}>C$ where $C$ is the constant appearing in (2.3). Define $E_{j}^{k}=Q_{j}^{k} \cap\left(\Omega_{k+m-1}-\Omega_{k+m}\right)$ for all $(k, j)$. For $x \in E_{j}^{k} \subset \Omega_{k+m-1}$, the maximum principle $(2.3)$ yields

$$
\begin{aligned}
T\left(\chi_{3 Q_{j}^{k}} f \mu\right)(x) & =T(f \mu)(x)-T\left(\chi_{\left(3 Q_{j}^{k}\right)^{c}} f \mu\right)(x) \\
& >2^{k+m-1}-C 2^{k}>2^{k+m-1}-2^{k+m-2}=2^{k+m-2} \geq 2^{k},
\end{aligned}
$$

and so

$$
\begin{aligned}
\left|E_{j}^{k}\right|_{\omega} & \leq 2^{-k} \int_{E_{j}^{k}} T\left(\chi_{3 Q_{j}^{k}} f \mu\right) d \omega=2^{-k} \int_{3 Q_{j}^{k}} f T\left(\chi_{E_{j}^{k}} \omega\right) d \mu \\
& =2^{-k}\left[\int_{3 Q_{j}^{k}-\Omega_{k+m}} f T\left(\chi_{E_{j}^{k}} \omega\right) d \mu+\int_{3 Q_{j}^{k} \cap \Omega_{k+m}} f T\left(\chi_{E_{j}^{k}} \omega\right) d \mu\right] \\
& =2^{-k}\left[\sigma_{j}^{k}+\tau_{j}^{k}\right] .
\end{aligned}
$$


We now estimate the left side of (1.4) by

$$
\begin{aligned}
\int[T(f \mu)]^{q} d \omega & \leq \sum_{k}\left(2^{k+m}\right)^{q}\left|\Omega_{k+m-1}-\Omega_{k+m}\right|_{\omega} \\
& \leq C \sum_{k, j}\left|E_{j}^{k}\right|_{\omega} 2^{k q} \\
& =C\left(\sum_{(k, j) \in E}+\sum_{(k, j) \in F}+\sum_{(k, j) \in G}\right)\left|E_{j}^{k}\right|_{\omega} 2^{k q} \\
& =C(\mathrm{I}+\mathrm{II}+\mathrm{III})
\end{aligned}
$$

where

$$
\begin{aligned}
& E=\left\{(k, j):\left|E_{j}^{k}\right|_{\omega} \leq \beta\left|Q_{j}^{k}\right|_{\omega}\right\}, \\
& F=\left\{(k, j):\left|E_{j}^{k}\right|_{\omega}>\beta\left|Q_{j}^{k}\right|_{\omega} \text { and } \sigma_{j}^{k}>\tau_{j}^{k}\right\}, \\
& G=\left\{(k, j):\left|E_{j}^{k}\right|_{\omega}>\beta\left|Q_{j}^{k}\right|_{\omega} \text { and } \sigma_{j}^{k} \leq \tau_{j}^{k}\right\},
\end{aligned}
$$

and where $\beta$, to be chosen later, satisfies $0<\beta<1$. We have

$$
\begin{aligned}
& \mathrm{I}=\sum_{(k, j) \in E}\left|E_{j}^{k}\right|_{\omega} 2^{k q} \leq \beta \sum_{k, j}\left|Q_{j}^{k}\right|_{\omega} 2^{k q} \\
& \leq \beta \sum_{k} 2^{k q}\left|\left\{T(f \mu)>2^{k}\right\}\right|_{\omega} \quad \text { by (2.2)(i) } \\
& \leq \beta \int\left(\sum_{k} 2^{k q} \chi_{\left\{T f \mu>2^{k}\right\}}\right) d \mu \\
& \leq C \beta \int[T(f \mu)]^{q} d \omega \\
& \mathrm{II}=\sum_{(k, j) \in F}\left|E_{j}^{k}\right|_{\omega} 2^{k q} \leq \sum_{(k, j) \in F}\left|E_{j}^{k}\right|_{\omega}\left[\frac{2 \sigma_{j}^{k}}{\left|E_{j}^{k}\right|_{\omega}}\right]^{q} \\
& \leq C \beta^{-q} \sum_{k, j}\left|E_{j}^{k}\right|_{\omega}\left[\frac{1}{\left|Q_{j}^{k}\right|_{\omega}} \int_{3 Q_{j}^{k}-\Omega_{k+m}} f T\left(\chi_{E_{j}^{k}} \omega\right) d \mu\right]^{q} \\
& \leq C \beta^{-q} \sum_{k, j} \frac{\left|E_{j}^{k}\right|_{\omega}}{\left|Q_{j}^{k}\right|_{\omega}^{q}}\left[\int_{3 Q_{j}^{k}}\left[T\left(\chi_{Q_{j}^{k}} \omega\right)\right]^{p^{\prime}} d \mu\right]^{q / p^{\prime}}\left[\int_{3 Q_{j}^{k}-\Omega_{k+m}} f^{p} d \mu\right]^{q / p} \\
& \leq C\left(C_{2}\right)^{q} \beta^{-q} \sum_{k, j}\left(\int_{3 Q_{j}^{k}-\Omega_{k+m}} f^{p} d \mu\right)^{q / p} \quad \text { by }(1.6) \\
& \leq C \beta^{-q}\left(\sum_{k, j} \int_{3 Q_{j}^{k}-\Omega_{k+m}} f^{p} d \mu\right)^{q / p} \quad \text { since } p \leq q \\
& \leq C \beta^{-q}\left(\int f^{p} d \mu\right)^{q / p}
\end{aligned}
$$


since

$$
\sum_{k, j} \chi_{3 Q_{j}^{k}-\Omega_{k+m}} \leq C \sum_{k} \chi_{\Omega_{k}-\Omega_{k+m}} \leq C(m+1) \quad \text { by }(2.2)(\mathrm{iii}) .
$$

To estimate term III in (2.4) we will need the following easy variant of the maximal theorem in [37]. For $\nu$ a positive Borel measure on $\mathbf{R}^{n}$, define

$$
M_{\nu} f(x)=\sup _{\substack{x \in Q \text { dyadic } \\ \text { cube }}}\left[\frac{1}{|Q|_{\nu}} \int_{Q}|f| d \nu\right], \quad f \in L_{\text {loc }}^{1}(\nu) .
$$

MAXIMAL ThEOREM. For $1<r \leq \infty$, and $\nu$ a positive Borel measure on $\mathbf{R}^{n}$,

$$
\int\left(M_{\nu} f\right)^{r} d \nu \leq C_{r} \int|f|^{r} d \nu \quad \text { for all } f \in L^{r}(\nu) .
$$

Inequality (2.7) follows by interpolation from the weak type $(1,1)$ and type $(\infty, \infty)$ estimates for $M_{\nu}$ (both with constant 1) as in [37].

Let $H_{j}^{k}=\left\{i: Q_{i}^{k+m} \cap 3 Q_{j}^{k} \neq \varnothing\right\}$ so that $3 Q_{j}^{k} \cap \Omega_{k+m} \subset \bigcup_{i \in H_{j}^{k}} Q_{i}^{k+m}$. In order to estimate $\tau_{j}^{k}$ we observe that the growth condition imposed on the kernel $K(x)$ implies that for $y \notin 3 Q_{i}^{k+m}$,

$$
\max _{x \in Q_{i}^{k+m}} K(x-y) \leq C \min _{x \in Q_{i}^{k+m}} K(x-y)
$$

which in turn yields

$$
\max _{x \in Q_{i}^{k+m}} T\left(\chi_{E_{j}^{k}} \omega\right)(x) \leq C \min _{x \in Q_{i}^{k+m}} T\left(\chi_{E_{j}^{k}} \omega\right)(x), \quad i \in H_{j}^{k},
$$

since $3 Q_{i}^{k+m} \subset \Omega_{k+m}$ (see $\left.(2.2)(\mathrm{ii})\right)$ and $\Omega_{k+m}$ does not intersect $E_{j}^{k}$. It follows that

$$
\begin{aligned}
\tau_{j}^{k} & =\int_{3 Q_{j}^{k} \cap \Omega_{k+m}} f T\left(\chi_{E_{j}^{k}} \omega\right) d \mu \leq C \sum_{i \in H_{j}^{k}}\left[\min _{x \in Q_{i}^{k+m}} T\left(\chi_{E_{j}^{k}} \omega\right)(x)\right] \int_{Q_{i}^{k+m}} f d \mu \\
& \leq C \sum_{i \in H_{j}^{k}}\left[\int_{Q_{i}^{k+m}} T\left(\chi_{E_{j}^{k}} \omega\right) d \mu\right]\left[\frac{1}{\left|Q_{i}^{k+m}\right|_{\mu}} \int_{Q_{i}^{k+m}} f d \mu\right] .
\end{aligned}
$$

For notational convenience, set

$$
A_{j}^{k}=\frac{1}{\left|Q_{j}^{k}\right|_{\mu}} \int_{Q_{j}^{k}} f d \mu
$$

and let $L_{j}^{k}=\left\{s: Q_{s}^{k} \cap 3 Q_{j}^{k} \neq \varnothing\right\}$. Then we have

$$
\begin{aligned}
\tau_{j}^{k} & \leq C \sum_{i \in H_{j}^{k}}\left[\int_{Q_{i}^{k+m}} T\left(\chi_{E_{j}^{k}} \omega\right) d \mu\right] A_{i}^{k+m} \\
& \leq C \sum_{s \in L_{j}^{k}}\left[\sum_{i: Q_{i}^{k+m} \subset Q_{s}^{k}}\left[\int_{Q_{i}^{k+m}} T\left(\chi_{E_{j}^{k}} \omega\right) d \mu\right] A_{i}^{k+m}\right] .
\end{aligned}
$$


For future reference, note that the cardinality of $L_{j}^{k}$ is at most $C$ by $(2.2)$ (iv). We now claim that

$$
\sum_{\substack{k, j) \in G \\ k \geq N \\ k(\bmod m)}}\left|E_{j}^{k}\right|_{\omega} 2^{k q} \leq C\left(\int f^{p} d \mu\right)^{q / p}
$$

with a constant $C$ independent of the integers $N$ and $M$ where $-\infty<N<\infty$, $0 \leq M<m$. Fix such integers $N$ and $M$ and introduce the convention, in force until the proof of $(2.10)$ is completed, that all indices $(k, j)$ are understood to be restricted to $k \geq N, k \equiv M(\bmod m)$.

With this convention we introduce "principal" cubes as in B. Muckenhoupt and R. L. Wheeden $\left[29\right.$, p. 804]. Let $G_{0}$ consist of those indices $(k, j)$ for which $Q_{j}^{k}$ is maximal. If $G_{n}$ has been defined, let $G_{n+1}$ consist of those $(k, j)$ for which there is $(t, u) \in G_{n}$ with $Q_{j}^{k} \subset Q_{u}^{t}$ and

(i) $A_{j}^{k}>2 A_{u}^{t}$,

(ii) $A_{i}^{l} \leq 2 A_{u}^{t}$ whenever $Q_{j}^{k} \varsubsetneqq Q_{i}^{l} \subset Q_{u}^{t}$.

Define $\Gamma=\bigcup_{n=0}^{\infty} G_{n}$ and for each $(k, j)$, define $P\left(Q_{j}^{k}\right)$ to be the smallest cube $Q_{u}^{t}$ containing $Q_{j}^{k}$ and with $(t, u) \in \Gamma$. Then we have

(i) $P\left(Q_{j}^{k}\right)=Q_{u}^{t}$ implies $A_{j}^{k} \leq 2 A_{u}^{t}$,

(ii) $Q_{j}^{k} \varsubsetneqq Q_{u}^{t},(k, j)$ and $(t, u) \in \Gamma$ imply $A_{j}^{k}>2 A_{u}^{t}$.

Using (2.9) and the fact that the cardinality of $L_{j}^{k}$ is at most $C$, we obtain (2.12)

$$
\begin{aligned}
& \sum_{(k, j) \in G}\left|E_{j}^{k}\right|_{\omega} 2^{k q} \leq \sum_{(k, j) \in G}\left|E_{j}^{k}\right|_{\omega}\left[\frac{2 \tau_{j}^{k}}{\left|E_{j}^{k}\right|_{\omega}}\right] \\
& \leq C \beta^{-q} \sum_{k, j} \frac{\left|E_{j}^{k}\right|_{\omega}}{\left|Q_{j}^{k}\right|_{\omega}^{q}}\left[\tau_{j}^{k}\right]^{q} \\
& \leq C \beta^{-q} \sum_{k, j} \sum_{s \in L_{j}^{k}} \frac{\left|E_{j}^{k}\right|_{\omega}}{\left|Q_{j}^{k}\right|_{\omega}^{q}}\left[\sum_{\substack{i: P\left(Q_{i}^{k+m}\right)=P\left(Q_{s}^{k}\right) \\
Q_{i}^{k+m} \subset Q_{s}^{k}}}\left[\int_{Q_{i}^{k+m}} T\left(\chi_{Q_{j}^{k}} \omega\right) d \mu\right] A_{i}^{k+m}\right]^{q} \\
& +C \beta^{-q} \sum_{k, j} \frac{\left|E_{j}^{k}\right|_{\omega}}{\left|Q_{j}^{k}\right|_{\omega}^{q}}\left[\sum_{i \in H_{j}^{k}:(k+m, i) \in \Gamma}\left[\int_{Q_{i}^{k+m}} T\left(\chi_{Q_{j}^{k}} \omega\right) d \mu\right] A_{i}^{k+m}\right]^{q} \\
& \text { (since if } Q_{i}^{k+m} \subset Q_{s}^{k} \text { and }(k+m, i) \notin \Gamma \text {, } \\
& \text { then } \left.P\left(Q_{i}^{k+m}\right)=P\left(Q_{s}^{k}\right)\right) \\
& =\mathrm{IV}+\mathrm{V} \text {. }
\end{aligned}
$$

We will use conditions (1.5) and (1.6) to estimate terms IV and V respectively. 
First, note that for a fixed $(t, u) \in \Gamma$,

$$
\begin{aligned}
& \sum_{k, j} \sum_{s \in L_{j}^{k}: P\left(Q_{s}^{k}\right)=Q_{u}^{t}} \frac{\left|E_{j}^{k}\right|_{\omega}}{\left|Q_{j}^{k}\right|_{\omega}^{q}}\left[\sum_{\begin{array}{c}
i: P\left(Q_{i}^{k+m}\right)=P\left(Q_{s}^{k}\right) \\
Q_{i}^{k+m} C Q_{s}^{k}
\end{array}}\left[\int_{Q_{i}^{k+m}} T\left(\chi_{Q_{j}^{k}} \omega\right) d \mu\right] A_{i}^{k+m}\right]^{q} \\
& \leq \sum_{k, j} \sum_{s \in L_{j}^{k}: P\left(Q_{s}^{k}\right)=Q_{u}^{t}}\left|E_{j}^{k}\right|_{\omega}\left[\frac{1}{\left|Q_{j}^{k}\right|_{\omega}} \int_{Q_{s}^{k}} T\left(\chi_{Q_{j}^{k}} \omega\right) d \mu\right]^{q}\left(2 A_{u}^{t}\right)^{q} \quad \text { by }(2.11)(\mathrm{i}) \\
& \leq\left(2 A_{u}^{t}\right)^{q} \sum_{k, j} \sum_{s \in L_{j}^{k}: P\left(Q_{s}^{k}\right)=Q_{u}^{t}}\left|E_{j}^{k}\right|_{\omega}\left[\frac{1}{\left|Q_{j}^{k}\right|_{\omega}} \int_{Q_{j}^{k}} T\left(\chi_{Q_{u}^{t}} \mu\right) d \omega\right]^{q} \\
& \leq C\left(A_{u}^{t}\right)^{q} \int\left[M_{\omega}\left[T\left(\chi_{Q_{u}^{t}} \mu\right)\right]\right]^{q} d \omega \quad \text { since cardinality of } L_{j}^{k} \leq C(n, R) \\
& \leq C\left(A_{u}^{t}\right)^{q} \int\left[T\left(\chi_{Q_{u}^{t}} \mu\right)\right]^{q} d \omega \quad \text { by the maximal theorem } \\
& \leq C\left(A_{u}^{t}\right)^{q}\left(C_{1}\right)^{q}\left|Q_{u}^{t}\right|_{\mu}^{q / p} \quad \text { by (1.5). }
\end{aligned}
$$

Summing (2.13) over $(t, u) \in \Gamma$ yields

$$
\mathrm{IV} \leq C \beta^{-q} \sum_{(t, u) \in \Gamma}\left|Q_{u}^{t}\right|_{\mu}^{q / p}\left(A_{u}^{t}\right)^{q} \leq C \beta^{-q}\left[\sum_{(t, u) \in \Gamma}\left|Q_{u}^{t}\right|_{\mu}\left(A_{u}^{t}\right)^{p}\right]^{q / p} \quad \text { since } p \leq q .
$$

To obtain the corresponding estimate for $V$, we note that for a fixed $(k, j)$, Hölder's inequality yields

$$
\begin{aligned}
& \frac{\left|E_{j}^{k}\right|_{\omega}}{\left|Q_{j}^{k}\right|_{\omega}^{q}}\left[\sum_{i \in H_{j}^{k}:(k+m, i) \in \Gamma}\left[\int_{Q_{i}^{k+m}} T\left(\chi_{Q_{j}^{k}} \omega\right) d \mu\right] A_{i}^{k+m}\right]^{q} \\
& \leq \frac{\left|E_{j}^{k}\right|_{\omega}}{\left|Q_{j}^{k}\right|_{\omega}^{q}}\left[\sum_{i \in H_{j}^{k}}\left|Q_{i}^{k+m}\right|_{\mu}^{-p^{\prime} / p}\left[\int_{Q_{i}^{k+m}} T\left(\chi_{Q_{j}^{k}} \omega\right) d \mu\right]^{p^{\prime}}\right]^{q / p^{\prime}} \\
& \times\left[\sum_{i \in H_{j}^{k}:(k+m, i) \in \Gamma}\left|Q_{i}^{k+m}\right|_{\mu}\left(A_{i}^{k+m}\right)^{p}\right]^{q / p} \\
& \leq \frac{\left|E_{j}^{k}\right|_{\omega}}{\left|Q_{j}^{k}\right|_{\omega}^{q}}\left[\sum_{i \in H_{j}^{k}} \int_{Q_{i}^{k+m}}\left[T\left(\chi_{Q_{j}^{k}} \omega\right)\right]^{p^{\prime}} d \mu\right]^{q / p^{\prime}}\left[\sum_{i \in H_{j}^{k}:(k+m, i) \in \Gamma}\left|Q_{i}^{k+m}\right|_{\mu}\left(A_{i}^{k+m}\right)^{p}\right]^{q / p} \\
& \leq\left(C_{2}\right)^{q}\left[\sum_{i \in H_{j}^{k}:(k+m, i) \in \Gamma}\left|Q_{i}^{k+m}\right|_{\mu}\left(A_{i}^{k+m}\right)^{p}\right]^{q / p} \quad \text { by }(1.6) .
\end{aligned}
$$


Summing (2.15) over $(k, j)$, using the fact that $p \leq q$, and then noting that any fixed $Q_{i}^{k+m}$ occurs at most $C$ times in the resulting sum (by (2.2)(iii)), we obtain

$$
V \leq C \beta^{-q}\left[\sum_{(t, u) \in \Gamma}\left|Q_{u}^{t}\right|_{\mu}\left(A_{u}^{t}\right)^{p}\right]^{q / p} .
$$

Combining (2.12), (2.14) and (2.16) shows that the left side of (2.10) is bounded by

$$
\begin{aligned}
& C \beta^{-q} {\left[\sum_{(t, u) \in \Gamma}\left|Q_{u}^{t}\right|_{\mu}\left(A_{u}^{t}\right)^{p}\right]^{q / p} } \\
& \leq C \beta^{-q}\left[\int\left[\sum_{(t, u) \in \Gamma}\left(A_{u}^{t}\right)^{p} \chi_{Q_{u}^{t}}(x)\right] d \mu(x)\right]^{q / p} \\
& \leq C \beta^{-q}\left[\int\left(M_{\mu} f\right)^{p} d \mu\right]^{q / p}
\end{aligned}
$$

since (2.11)(ii) implies that for any fixed $x$,

$$
\sum_{(t, u) \in \Gamma}\left(A_{u}^{t}\right)^{p} \chi_{Q_{u}^{t}}(x) \leq 2^{p} \sup _{x \in Q_{u}^{t}}\left(A_{u}^{t}\right)^{p} \leq 2^{p} M_{\mu} f(x)^{p} .
$$

From (2.17) and the maximal theorem we obtain (2.10). Now let $N \rightarrow-\infty$ in (2.10) and then sum over $M=0,1,2, \ldots, m-1$ to obtain

$$
\mathrm{III} \leq C \beta^{-q}\left(\int f^{p} d \mu\right)^{q / p} .
$$

Combining (2.4), (2.5), (2.6) and (2.18) we have

$$
\int[T(f \mu)]^{q} d \omega \leq C \beta \int[T(f \mu)]^{q} d \omega+C \beta^{-q}\left(\int f^{p} d \mu\right)^{q / p} .
$$

Now chose $\beta$ so small that $C \beta<\frac{1}{2}$ and then subtract the first term on the right side of (2.19) from both sides (it is finite by (1.5) and our assumptions on $f$ ) to obtain (1.4) for $f \geq 0$ bounded with compact support, and hence for arbitrary $f \geq 0$ by the monotone convergence theorem. This completes the proof of Theorem 1 .

3. Proof of Theorem 2. The proof of Theorem 2 follows very closely the line of argument used in Theorem 1, but applied to the dual Poisson operator $P^{*}$ rather than $P$. In order to minimize confusion in referring to the proof of Theorem 1 , we set $T=P^{*}$, i.e. $T(f \mu)(x)=\int P_{t}(x-y) f(y, t) d \mu(y, t)$, interchange the roles of $\omega$ and $\mu, q$ and $p^{\prime}$, and consider instead the inequality,

$$
\left(\int_{\mathbf{R}^{n}}[T(f \mu)]^{q} d \omega\right)^{1 / q} \leq C\left(\int_{\mathbf{R}_{+}^{n+1}} f^{p} d \mu\right)^{1 / p} \quad \text { for all } f \geq 0 .
$$


We will show that (3.1) holds if and only if both

$$
\begin{gathered}
{\left[\int_{\mathbf{R}^{n}}\left(T\left[\chi_{\hat{Q}}(x, t) t^{p^{\prime}-1} d \mu(x, t)\right]\right)^{q} d \omega\right]^{1 / q} \leq C\left[\int_{\hat{Q}} t^{p^{\prime}} d \mu(x, t)\right]^{1 / p}<\infty} \\
{\left[\int_{\mathbf{R}_{+}^{n+1}}\left(P \chi_{Q} \omega\right)^{p^{\prime}} d \mu\right]^{1 / p^{\prime}} \leq C\left(\int_{Q} d \omega\right)^{1 / q^{\prime}}<\infty}
\end{gathered}
$$

hold for all dyadic cubes $Q \subset \mathbf{R}^{n}$.

Condition (3.2) follows by setting $f(x, t)=\chi_{\hat{Q}}(x, t) t^{p^{\prime}-1}$ in (3.1), and (3.3) follows by testing the inequality dual to (3.1) with $\chi_{Q}$.

Conversely, we begin exactly as in the proof of Theorem 1 but with the maximum principle in (2.3) replaced by

$$
T\left(\chi_{\left(3 \hat{Q}_{j}^{k}\right)^{c}} f \mu\right)(x) \leq C 2^{k}, \quad x \in Q_{j}^{k} .
$$

To see (3.4), choose $z \in 3 R Q_{j}^{k} \cap \Omega_{k}^{c}$ as before by the Whitney condition. Inequality (3.4) now follows from the inequality

$$
P_{t}(x-y) \leq C P_{t}(z-y), \quad x \in Q_{j}^{k},(y, t) \notin 3 \hat{Q}_{j}^{k},
$$

after multiplying by $f(y, t)$ and then integrating over $\left(3 \hat{Q}_{j}^{k}\right)^{c}$ with respect to $d \mu(y, t)$.

From this point up to the inequality in (2.8), the proof of Theorem 1 can be applied verbatim provided that in the context of the measure space $\left(\mathbf{R}_{+}^{n+1}, d \mu\right)$, cubes $Q$ are replaced by $\hat{Q}$ and the sets $\Omega_{k}$ are replaced by $\hat{\Omega}_{k}=\bigcup_{j} \hat{Q}_{j}^{k}$. The only new development in this proof arises now: $T^{*}\left(\chi_{E_{j}^{k}} \omega\right)$ is no longer roughly constant on any $\hat{Q}_{i}^{k+m}$, but merely roughly constant on level planes of $\hat{Q}_{i}^{k+m}$ (see (3.6) below). The substitute for (2.8) is

$$
\begin{aligned}
\tau_{j}^{k} & =\int_{3 \hat{Q}_{j}^{k} \cap \hat{\Omega}_{k+m}} f T^{*}\left(\chi_{E_{j}^{k}} \omega\right) d \mu=\sum_{i \in H_{j}^{k}} \int_{\hat{Q}_{i}^{k+m}} f T^{*}\left(\chi_{E_{j}^{k}} \omega\right) d \mu \\
& \leq C \sum_{i \in H_{j}^{k}}\left[\int_{\hat{Q}_{i}^{k+m}} T^{*}\left(\chi_{E_{j}^{k}} \omega\right) t^{-1} d \tilde{\mu}\right]\left[\frac{1}{\left|\hat{Q}_{i}^{k+m}\right|_{\tilde{\mu}}} \int_{\hat{Q}_{i}^{k+m}} f t^{1-p^{\prime}} d \tilde{\mu}\right]
\end{aligned}
$$

where $d \tilde{\mu}(y, t)=t^{p^{\prime}} d \mu(y, t)$. To see the inequality in (3.5), observe that if $x_{i}^{k+m}$ is the centre of $Q_{i}^{k+m}$, then for $(x, t) \in \hat{Q}_{i}^{k+m}$,

$$
T^{*}\left(\chi_{E_{j}^{k}} \omega\right)(x, t) \approx T^{*}\left(\chi_{E_{j}^{k}} \omega\right)\left(x_{i}^{k+m}, t\right) \approx \frac{t}{d_{j}^{k}} T^{*}\left(\chi_{E_{j}^{k}} \omega\right)\left(x_{i}^{k+m}, d_{j}^{k}\right)
$$

since $3 Q_{i}^{k+m} \cap E_{j}^{k}=\varnothing$. Here $d_{j}^{k}$ is the side length of $Q_{j}^{k}$ and the symbol of approximate equality means the ratio of the sides is bounded between absolute 
positive constants. From (3.6) we have

$$
\begin{array}{rl}
\int_{\hat{Q}_{i}^{k+m}} & f T^{*}\left(\chi_{E_{j}^{k}} \omega\right) d \mu \approx \frac{1}{d_{j}^{k}} T^{*}\left(\chi_{E_{j}^{k}} \omega\right)\left(x_{i}^{k+m}, d_{j}^{k}\right) \int_{\hat{Q}_{i}^{k+m}} f(x, t) t d \mu(x, t) \\
= & {\left[\frac{\int_{\hat{Q}_{i}^{k+m}} \frac{t}{d_{j}^{k}} T^{*}\left(\chi_{E_{j}^{k}} \omega\right)\left(x_{i}^{k+m}, d_{j}^{k}\right) t^{p^{\prime}-1} d \mu(x, t)}{\left.\int_{\hat{Q}_{i}^{k+m} t^{p^{\prime}} d \mu(x, t)}\right] \int_{\hat{Q}_{i}^{k+m}} f(x, t) t d \mu(x, t)}\right.} \\
\approx & {\left[\frac{\int_{\hat{Q}_{i}^{k+m}} T^{*}\left(\chi_{E_{j}^{k}} \omega\right)(x, t) t^{-1} d \tilde{\mu}(x, t)}{\int_{\hat{Q}_{i}^{k+m}} d \tilde{\mu}}\right] \int_{\hat{Q}_{i}^{k+m}} f t^{1-p^{\prime}} d \tilde{\mu}}
\end{array}
$$

which yields (3.5) upon summing over $i \in H_{j}^{k}$.

In view of (3.5), it is now appropriate to define the averages $A_{j}^{k}$ by $\left(1 /\left|\hat{Q}_{j}^{k}\right|_{\tilde{\mu}}\right) \int_{\hat{Q}_{j}^{k}} f t^{1-p^{\prime}} d \tilde{\mu}$. With these changes, the argument in the proof of Theorem 1 now leads to the conclusion, as in (2.17), that the left side of (2.10) is dominated by $C \beta^{-q}\left(\int\left(M_{\tilde{\mu}} g\right)^{p} d \tilde{\mu}\right)^{q / p}$ where $g(x, t)=t^{1-p^{\prime}} f(x, t)$ and

$$
M_{\tilde{\mu}} g(x, t)=\sup _{\substack{(x, t) \in Q \text { dyadic } \\ \text { cube in } R_{+}^{n+1}}}\left[\frac{1}{|Q|_{\tilde{\mu}}} \int_{Q}|g| d \tilde{\mu}\right] .
$$

The maximal theorem now shows that

$$
\begin{aligned}
\int\left(M_{\tilde{\mu}}\right)^{p} d \tilde{\mu} & \leq C \int g^{p} d \tilde{\mu}=C \int f^{p} t^{\left(1-p^{\prime}\right) p} t^{p^{\prime}} d \mu \\
& =C \int f^{p} d \mu
\end{aligned}
$$

and the proof of Theorem 2 is now completed as in the proof of Theorem 1.

\section{REFERENCES}

1. M. Artola, untitled and unpublished manuscript.

2. A. Bonami and R. Johnson, Tent spaces based on the Lorentz spaces (to appear).

3. J. S. Bradley, Hardy inequalities with mixed norms, Canad. Math. Bull. 21 (1978), 405-408.

4. L. Carleson, An interpolation problem for bounded analytic functions, Amer. J. Math. 80 (1958), 921-930.

5. S. Y. A. Chang, J. Wilson and T. Wolff, Some weighted norm inequalities concerning the Schrodinger operators, Comment. Math. Helv. 60 (1985), 217-246.

6. S. Chanillo and R. L. Wheeden, Weighted Poincaré and Sobolev inequalities and estimates for weighted Peano maximal functions, Amer. J. Math. 107 (1985), 1191-1226.

7. $-L^{p}$ estimates for fractional integrals and Sobolev inequalities with applications to Schrodinger operators, Comm. Partial Differential Equations 10 (1985), 1077-1116.

8. , Harnack's inequality and mean-value inequalities for solutions of degenerate elliptic equations (to appear).

9. M. Cotlar and C. Sadosky, On some $L^{p}$ versions of the Helson-Szego theorem, Conf. Harmonic Analysis in honor of Antoni Zygmund, Wadsworth Math. Series, Wadsworth, Belmont, Calif., 1983.

10. B. E. J. Dahlberg, Regularity properties of Riesz potentials, Indiana Univ. Math. J. 28 (1979), 257-268.

11. P. L. Duren, Extension of a theorem of Carleson, Bull. Amer. Math. Soc. 75 (1969), 143-146.

12. E. B. Fabes, C. Kenig and R. Serapioni, The local regularity of solutions of degenerate elliptic equations, Comm. Partial Differential Equations 7 (1982), 77-116. 
13. C. Fefferman, Inequalities for strongly singular convolution operators, Acta Math. 124 (1970), 9-36.

14. __ The uncertainty principle, Bull. Amer. Math. Soc. (N.S.) 10 (1983), 129-206.

15. A. E. Gatto, C. E. Gutierrez and R. L. Wheeden, Fractional integrals on weighted $H^{p}$ spaces, Trans. Amer. Math. Soc. 289 (1985), 575-589.

16. M. de Guzman, Differentiation of integrals in $\mathbf{R}^{n}$, Lecture Notes in Math., vol. 481, SpringerVerlag, Berlin, 1975.

17. K. Hansson, Embedding theorems of Sobolev type in potential theory, Math. Scand. 45 (1979), 77-102.

18. E. Harboure, Two-weighted Sobolev and Poincare inequalities and some applications (to appear).

19. R. Hunt, B. Muckenhoupt and R. L. Wheeden, Weighted norm inequalities for the conjugate function and Hilbert transform, Trans. Amer. Math. Soc. 176 (1973), 227-241.

20. B. Jawerth, Weighted inequalities for maximal operators: linearization, localization and factorization, Amer. J. Math. (to appear).

21. R. Kerman and E. Sawyer, Weighted norm inequalities for potentials with applications to Schrodinger operatorss, Fourier transforms and Carleson measures, Bull. Amer. Math. Soc. (N.S.) 12 (1985), 112-116.

22. _ The trace inequality and eigenvalue estimates for Schrodinger operators, Ann. Inst. Fourier (Grenoble) 36 (1986), 207-228.

23. __ Carleson measures and multipliers for Dirichlet-type spaces, Trans. Amer. Math. Soc. (to appear).

24. B. Muckenhoupt, Hardy's inequality with weights, Studia Math. 44 (1972), 31-38.

25. __ Weighted norm inequalities for the Hardy maximal function, Trans. Amer. Math. Soc. 165 (1972), 207-226.

26. __ Weighted norm inequalities for classical operators, Proc. Sympos. Pure Math., vol. 35, part 1, Amer. Math. Soc., Providence, R.I., 1979, pp. 69-83.

27. B. Muckenhoupt and R. L. Wheeden, Weighted norm inequalities for fractional integrals, Trans. Amer. Math. Soc. 192 (1974), 261-274.

28. Hilbert transform, Studia Math. 55 (1976), 279-294.

29. __ Some weighted weak-type inequalities for the Hardy-Littlewood maximal function and the Hilbert transform, Indiana Univ. Math. J. 26 (1977), 801-816.

30. F. J. Ruiz Blasco and J. L. Torrea Hernandez, Weighted and vector valued inequalities for potential operators, Trans. Amer. Math. Soc. 295 (1986), 213-232.

31. E. T. Sawyer, A characterization of a two-weight norm inequality for maximal operators, Studia Math. 75 (1982), 1-11.

32. __ A two weight weak type inequality for fractional integrals, Trans. Amer. Math. Soc. 281 (1984), 339-345.

33. _ Weighted inequalities for the two-dimensional Hardy operator, Studia Math. 82 (1985), 1-16.

34. _, A weighted inequality and eigenvalue estimates for Schrodinger operators, India Univ. Math. J. 35 (1986), 1-28.

35. M. Schechter, Weighted norm estimates for Riesz potentials (to appear).

36. D. A. Stegenga, Multipliers of the Dirichlet space, Illinois J. Math. 24 (1980), 113-139.

37. E. M. Stein, Singular integrals and differentiability properties of functions, Princeton Univ. Press, Princeton, N.J., 1970.

38. E. Stredulinsky, Weighted inequalities and degenerate elliptic partial differential equations, Lecture Notes in Math., vol. 1074, Springer, 1984.

39. J.-0. Stromberg and R. L. Wheeden, Fractional integrals on weighted $H^{p}$ and $L^{p}$ spaces, Trans. Amer. Math. Soc. 287 (1985), 293-321.

40. G. Talenti, Osservazioni sopra una classe di disuguaglianze, Rend. Sem. Mat. Fis. Milano 39 (1969), 171-185.

41. G. Tomaselli, A class of inequalities, Boll. Un. Mat. Ital. 21 (1969), 622-631.

Department of Mathematics and Statistics, MCMaster University, HamilTON, ONTARIO, CANADA 